\title{
BMJ Open Control strategies to prevent total hip replacement-related infections: a systematic review and mixed treatment comparison
}

\author{
Henry Zheng, ${ }^{1}$ Adrian G Barnett, ${ }^{1}$ Katharina Merollini, ${ }^{1}$ Alex Sutton, ${ }^{2}$ \\ Nicola Cooper, ${ }^{2}$ Tony Berendt, ${ }^{3}$ Jennie Wilson, ${ }^{4}$ Nicholas Graves ${ }^{1}$
}

To cite: Zheng $H$, Barnett $A G$, Merollini K, et al. Control strategies to prevent total hip replacement-related infections: a systematic review and mixed treatment comparison. BMJ Open 2014:4:e003978. doi:10.1136/bmjopen-2013003978

- Additional material is available. To view please visit the journal (http://dx.doi.org/ 10.1136/bmjopen-2013003978).

Received 12 September 2013 Revised 10 January 2014 Accepted 2 February 2014

CrossMark

\footnotetext{
${ }^{1}$ Australian Centre for Health Services Innovation, Queensland University of Technology, Brisbane, Queensland, Australia ${ }^{2}$ Department of Health Sciences, University of Leicester, Leicester, UK ${ }^{3}$ Oxford University Hospitals NHS Trust, Oxford, UK

${ }^{4}$ Institute of Practice, Interdisciplinary Research and Enterprise, University of Western London, London, UK

Correspondence to Professor Nicholas Graves; n.graves@qut.edu.au
}

\section{ABSTRACT}

Objective: To synthesise the available evidence and estimate the comparative efficacy of control strategies to prevent total hip replacement (THR)-related surgical site infections (SSIs) using a mixed treatment comparison.

Design: Systematic review and mixed treatment comparison.

Setting: Hospital and other healthcare settings.

Participants: Patients undergoing THR.

Primary and secondary outcome measures:

The number of THR-related SSIs occurring following the surgical operation.

Results: 12 studies involving 123788 THRs and 9 infection control strategies were identified. The strategy of 'systemic antibiotics+antibiotic-impregnated cement +conventional ventilation' significantly reduced the risk of THR-related SSI compared with the referent strategy (no systemic antibiotics+plain cement+conventional ventilation), OR 0.13 ( $95 \%$ credible interval (Crl) $0.03-$ $0.35)$, and had the highest probability $(47-64 \%)$ and highest median rank of being the most effective strategy. There was some evidence to suggest that 'systemic antibiotics+antibiotic-impregnated cement +laminar airflow' could potentially increase infection risk compared with 'systemic antibiotics+antibioticimpregnated cement+conventional ventilation', 1.96 (95\% Crl 0.52-5.37). There was no high-quality evidence that antibiotic-impregnated cement without systemic antibiotic prophylaxis was effective in reducing infection compared with plain cement with systemic antibiotics, 1.28 (95\% Crl 0.38-3.38).

Conclusions: We found no convincing evidence in favour of the use of laminar airflow over conventional ventilation for prevention of THR-related SSIs, yet laminar airflow is costly and widely used. Antibiotic-impregnated cement without systemic antibiotics may not be effective in reducing THR-related SSIs. The combination with the highest confidence for reducing SSIs was 'systemic antibiotics+antibiotic-impregnated cement+conventional ventilation'. Our evidence synthesis underscores the need to review current guidelines based on the available evidence, and to conduct further high-quality doubleblind randomised controlled trials to better inform the current clinical guidelines and practice for prevention of THR-related SSIs.

\section{Strengths and limitations of this study}

This study is the first to examine the comparative effectiveness of various infection control strategies involving multiple infection control measures in preventing THR-related SSIs. Multiple sensitivity analyses contributed to the methodological rigour of the study.

- The small number of studies available for evidence synthesis reduced the statistical power and resulted in wide credible intervals for some comparisons.

- Owing to limited data available, the MTC model was unable to adjust for potential confounders such as casemix, different types of laminar airflow systems and temporal changes in clinical practices and infection control technology which may have taken place over the past several decades.

\section{INTRODUCTION}

Despite numerous advances in hip arthroplasty, surgical site infection (SSI) following total hip replacement (THR) remains a serious threat. Infection causes functional impairment, reduces quality of life and creates large costs for patients and the healthcare system. Identifying evidence based and effective infection control strategies to prevent THR-related SSI is critically important.

Evidence for the effectiveness of infection control measures in reducing THR-related SSI has been inconsistent. ${ }^{1-3}$ Previous evidence syntheses focused on single infection control measures such as systemic antibiotic prophylaxis, ${ }^{4}{ }^{5}$ antibiotic-impregnated cement ${ }^{6}{ }^{7}$ or ventilation systems alone ${ }^{8}$ without examining the combined effect of multiple control measures. In practice, infection control strategies combine multiple infection control measures, yet no good evidence is available on the combined comparative effectiveness of strategies involving multiple measures. 
Previous evidence syntheses relied on narrative systematic reviews or conventional pairwise meta-analysis. These do not compare the effectiveness of all trialled control measures when the evidence base of published studies does not include all possible comparisons. ${ }^{9}$ The remedy is to define a connected network of the evidence base and combine all the available data in a single mixed treatment comparison (MTC) model. ${ }^{9} 10$ This enables comparisons of all available infection control strategies to better inform decision making.

We conducted a MTC, also known as network meta-analysis, to synthesise the available evidence and determine the combined comparative effectiveness of infection control strategies in preventing THR-related SSI in patients undergoing THR.

\section{METHODS}

We applied the Patient, Intervention, Comparison and Outcome (PICO) framework. The population of interest was patients undergoing THR. The interventions were infection control strategies to prevent THR-related SSI. The comparison was an intervention strategy that was compared with the other intervention strategies in the MTC network. The outcome of interest was the number of THR-related SSIs. The PICO framework was specified in box 1 .

\section{Study identification}

We chose antibiotic prophylaxis, antibiotic-impregnated cement and laminar airflow based on published guidelines and a survey of expert opinion. ${ }^{11}$ We followed the systematic review guidelines in the PRISMA statement. ${ }^{12}$ We used a two-stage search strategy. First, we used systematic reviews by Glenny and Song ${ }^{4}$ and AlBuhairan et a $\check{l}^{\tilde{L}}$ to locate studies on the efficacy of systemic antibiotic prophylaxis in preventing THR-related infection. Together, these covered the years from 1966 to 2007. Systematic reviews by Parvizi et $a t^{6}$ and Block and Stubbs ${ }^{7}$ were used to locate trials on the effect of antibioticimpregnated cement in preventing THR-related SSI. These reviews covered the years from 1966 to 2004. We used the recent systematic review by Whitehead $e t a l^{8}$ to locate studies on the efficacy of operating theatre ventilation systems in preventing THR-related SSI, which covered the years from 1970 to 2007 .

\section{Box 1 Patient, Intervention, Comparison and Outcome} framework

Population: Patients undergoing total hip replacement. Intervention: Infection control strategies to prevent total hip replacement (THR)-related surgical site infection (SSI). Comparison: An infection control strategy compared with other control strategies in the mixed treatment comparison network. Outcome: The number of THR-related SSIs.
Second, we updated these systematic reviews by extending the search periods to June 2011. The electronic databases searched were MEDLINE, EMBASE, CINAHL and the Cochrane Central Register of Controlled Trials. Relevant journals, conference proceedings and bibliographies of retrieved papers were searched. Eleven orthopaedic surgeons and infection control experts from six hospitals were consulted. The search was limited to English-language papers (the search details are in online supplementary appendix 1).

Studies were included if they reported THR-related deep SSI or infection requiring a joint revision procedure as an outcome. While the precise definitions varied, they encompassed signs of infection involving the joint and/or fascial tissue at the site of the joint. Owing to the limited number of studies available, we included observational studies as well as randomised controlled trials (RCTs).

Studies were excluded if THR-related SSIs were not separated from knee or other joint replacement-related infections. Studies that only compared different types, doses or durations of antibiotic regimens were treated as one-arm trials and excluded from the network meta-analysis as MTC relies on there being at least two arms that can become part of the network. ${ }^{10}$ The antibiotics were combined because there is little evidence of different efficacies in preventing THR-related SSI between antibiotics according to their type, dose or duration. ${ }^{4}$ The two-stage search process is in the flow chart (figure 1; reasons for exclusion are shown in online supplementary appendix 2).

\section{Data extraction}

Data were extracted by two independent reviewers and discrepancies were resolved by consensus. The key data were the number of THRs performed and THR-related deep SSIs; use of antibiotic prophylaxis and its delivery mode; and operating theatre ventilation system.

\section{Quality assessment}

The quality of the included studies and their level of evidence were assessed based on the National Institute for Health and Care Excellence (NICE) public health guidelines $^{13}$ (see online supplementary appendix 3), and quality scoring systems for RCTs by the Joanna Briggs Institute ${ }^{14}$ and observational longitudinal studies by Tooth $e t a l^{15}$ (see online supplementary appendix 4).

\section{Statistical methods}

MTC models produce estimates of the relative effects of each infection control strategy compared with every other strategy in a network, thus allowing coherent judgements to be made on which strategy is the most effective. ${ }^{9}$ It enables simultaneous comparisons of multiple infection control strategies from trials that did not necessarily directly compare all strategies. ${ }^{9}{ }^{10}$ Bayesian methods have been developed for MTC models (see online supplementary appendix 5). ${ }^{16}$ 


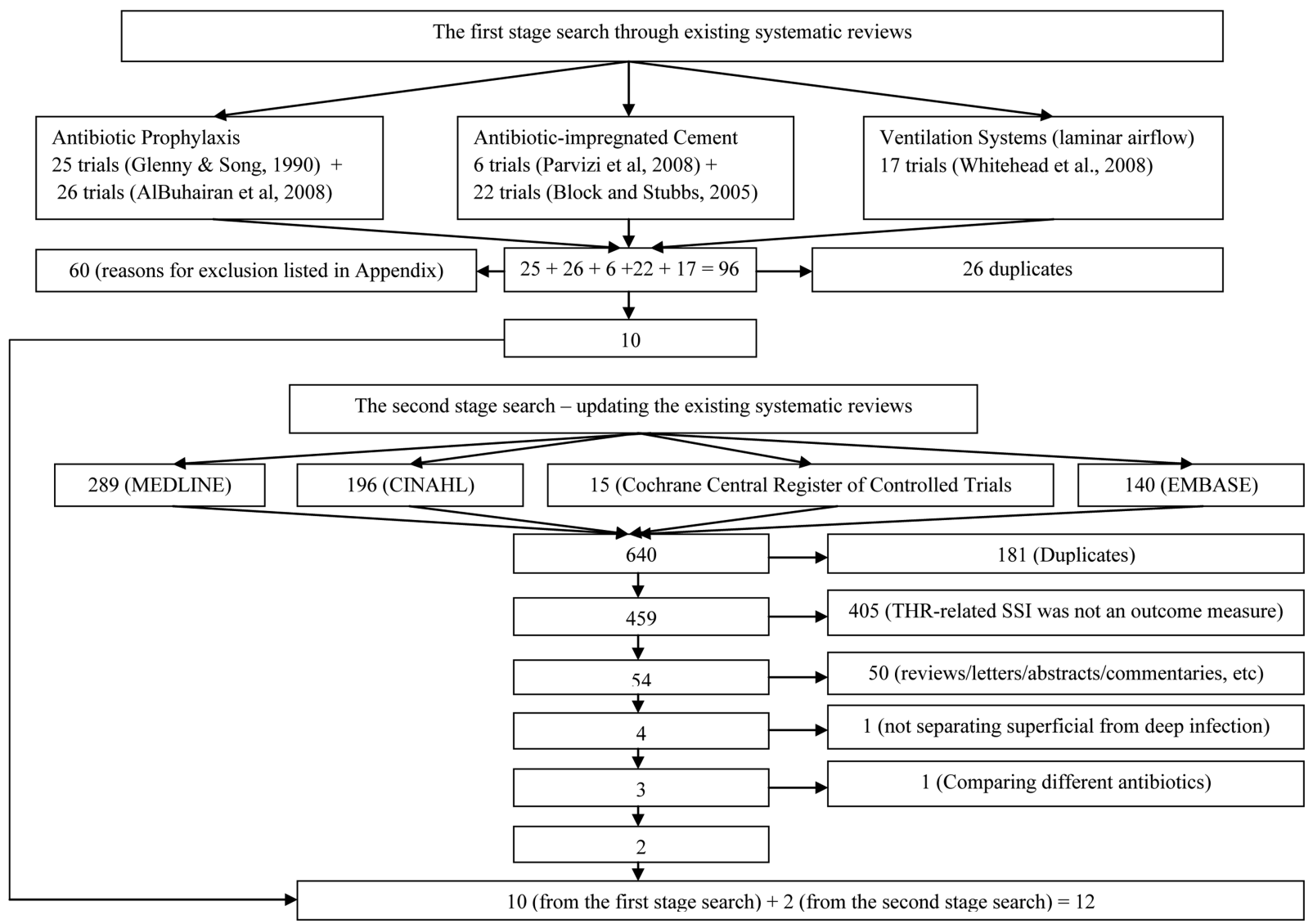

Figure 1 Two-stage literature search flow chart.

The MTC analysis was performed using a binomial random effect model allowing multiarm trials. ${ }^{17}$ The key summary statistics were the relative infection control effects using ORs, and the probability and median rank of being the most effective strategy. Studies with longer follow-up periods were likely to find more infections; hence, we accounted for this by modelling the duration of follow-up (see online supplementary appendix 6). The models were fitted in a Bayesian framework using the WinBUGS program and code by Dias et al. ${ }^{17}$

\section{Evaluation of model fit and evidence consistency}

We assessed the models' goodness of fit (see online supplementary appendix 7). Where the model fit was poor, we explored the influence of each study on the model fit (see online supplementary appendix 8).

An assumption of MTC models is that direct and indirect sources of evidence estimate the same true treatment effect across the network. We checked this assumption by conventional pairwise meta-analyses and by removing the constraint that direct and indirect evidence estimate the same effect. ${ }^{18}$ The latter is also known as nodesplitting (see online supplementary appendix 9 ).

\section{Heterogeneity and sensitivity analysis}

Heterogeneity of the MTC network was quantified by using the between-study SD. We performed sensitivity analyses by removing outliers as identified through diagnostic assessment.

There may have been a difference in evidence between RCTs and observational studies. To examine this, we performed a meta-regression with study type as an interaction (see online supplementary appendix 10) ${ }^{19}$ In further sensitivity analyses, we excluded the RCT by Hill $e t a l^{20}$ due to its reported violation of the RCT trial code, and included the RCT by Lidwell et al which was initially excluded because it did not separate THRs from knee replacements.

\section{RESULTS}

The two-stage search strategy yielded 529 studies, of which 12 met our inclusion criteria. Six were RCTs ${ }^{20-25}$ and six were observational studies. ${ }^{2}{ }^{3}{ }^{26-29}$ The studies included 123788 THRs and 9 infection control strategies as mapped in the MTC network (figure 2). The raw data are in the Summary of Evidence (table 1).

The quality of evidence was mixed with the level of evidence ranging from 1 to 2 (table 1 ).

Five of six studies ${ }^{21-25}$ provided no information on random sequence generation; four ${ }^{22-25}$ provided no information on blinding assessors; and only one reported prior calculation of the sample size. ${ }^{20}$ The statistical power for most RCTs was generally low. Only one 


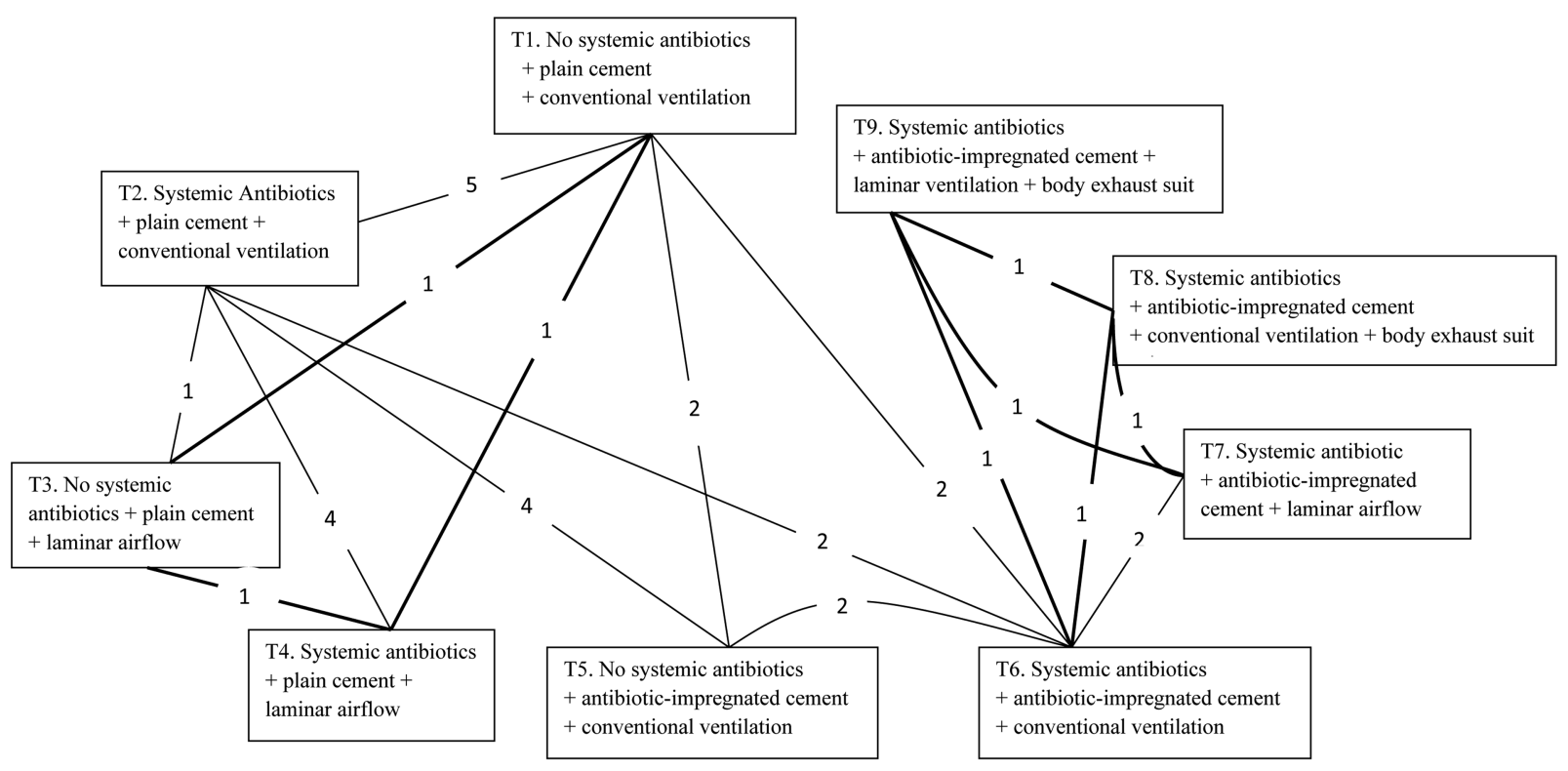

Note: The lines represent direct evidence comparisons; boxes represent infection control strategies involving multiple infection control measures; the numbers on the lines represent the numbers of comparisons. The three-way loops in bold lines represent loops only formed by a multi-arm trial

Figure 2 The mixed treatment comparison network consisting of 12 studies with 9 infection control strategies.

RCT reported primary analysis based on all randomised cases $^{20}$ while the rest did not report intention to treat.

Of the six observational studies, three ${ }^{328} 29$ identified and adjusted for confounding variables. One ${ }^{26}$ reported that cases and control groups were comparable on diagnostic confounding factors, and two ${ }^{28} 29$ described and included in the analysis the outcomes of the patients who withdrew. Four studies ${ }^{2} 32829$ used objective measures to assess the outcomes, and were adequately powered with large sample size ranging from 10905 to 51485 .

For every infection control strategy in the connected network, a relative effect was estimated against another infection control strategy using the OR. We chose 'no systemic antibiotics, plain cement and conventional ventilation' as the referent strategy, as it was compared with the greatest number of other strategies.

Thirty-six relative effects involving nine infection control strategies were estimated in the MTC network using models that did and did not adjust for duration of follow-up (see online supplementary appendix 11 and table 2). The results from both models were almost identical, as were estimates of the model fit. Hence, the differences in follow-up duration had little effect on the effectiveness of the infection strategies. We therefore report the results of the model without adjustment for follow-up from now on (table 2). The 36 ORs for all pairwise comparisons are in the Forest Plot (figure 3).

The five infection control strategies associated with a statistically significant reduction in THR-related SSI compared with the referent strategy T1 were: T6 (systemic antibiotics+antibiotic-impregnated cement+conventional ventilation), OR 0.13 (95\% credible interval (CrI) $0.03-$ 0.35); T2 (systemic antibiotics+plain cement+conventional ventilation), 0.31 (0.12-0.65); T3 (no systemic antibiotics +plain cement+laminar airflow), 0.26 (0.03-0.95); T4 (systemic antibiotics+plain cement+laminar airflow), 0.25 (0.06-0.66); and T7 (systemic antibiotics+antibioticimpregnated cement+laminar airflow), 0.27 (0.03-0.93; table 3).

Statistically non-significant reductions in THR-related SSIs as compared with the referent were T5 (no systemic antibiotics+antibiotic-impregnated cement+conventional ventilation), OR 0.38 (95\% CrI 0.09-1.12); T8 (systemic antibiotics+antibiotic-impregnated cement+conventional ventilation+body exhaust suit), $0.52(0.03-2.12)$ and T9 (systemic antibiotics+antibiotic-impregnated cement+ laminar ventilation+body exhaust suit), 0.74 (0.05-2.69).

The OR for T7 (systemic antibiotics+ antibioticimpregnated cement+laminar airflow) compared with T6 (systemic antibiotics+antibiotic-impregnated cement +conventional ventilation) was 1.96 (95\% CrI 0.52-5.37), suggesting that laminar airflow could potentially increase infection risk.

There was no high-quality evidence that antibioticimpregnated cement without systemic antibiotics was effective in reducing infection compared with plain cement with systemic antibiotics (T2 vs T5), 1.28 (95\% CrI 0.38-3.38).

Strategy T6 had the highest probability and highest median rank of being the best strategy in reducing THR-related SSI (see online supplementary appendix 12).

\section{Model fit and evidence consistency}

The model fit statistics indicated that the fit was less than adequate (table 2). This was confirmed by diagnostic plots, which showed that infection control strategies 
Table 1 Summary of evidence: comparisons of nine control strategies across the MTC network

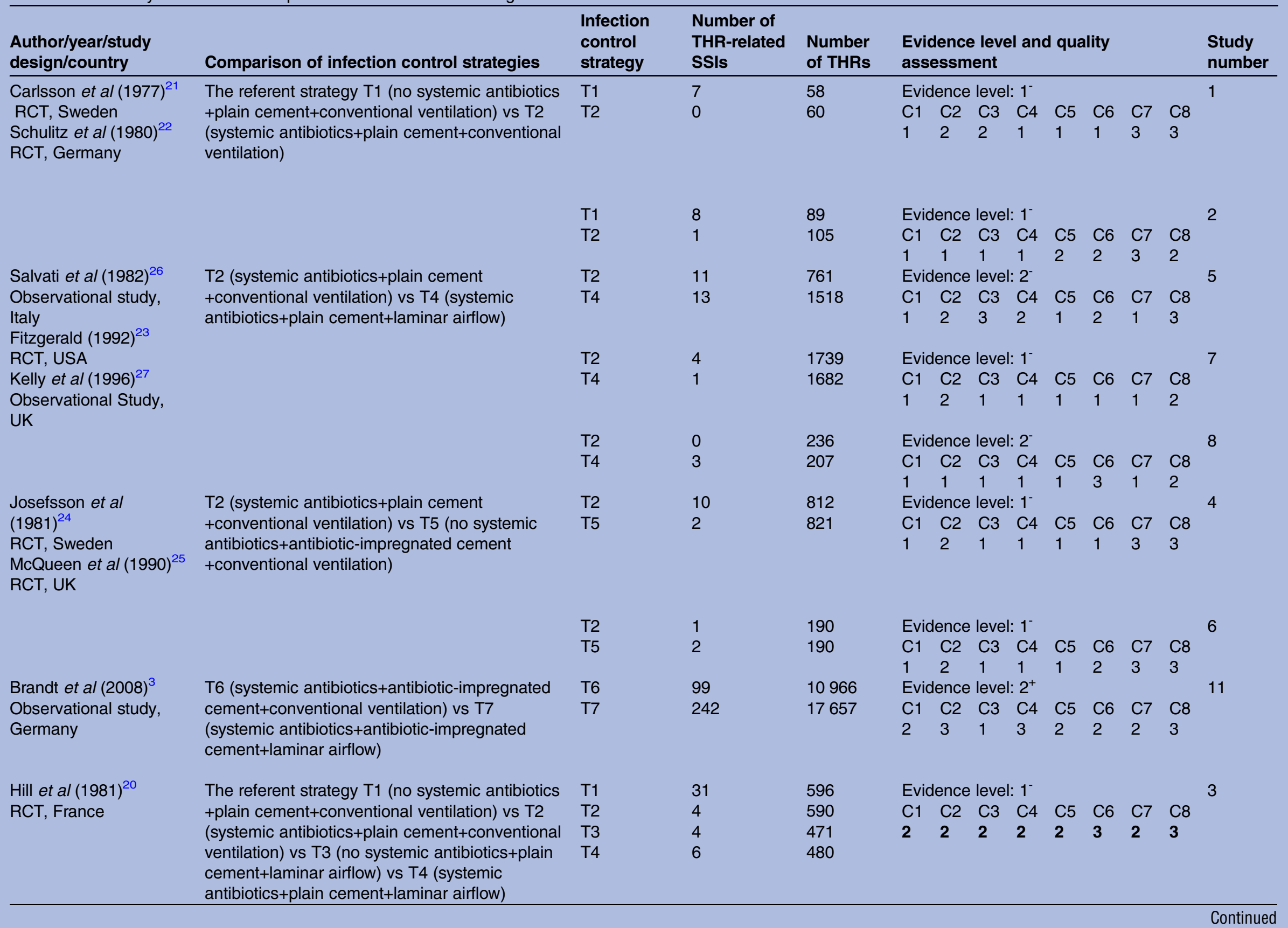




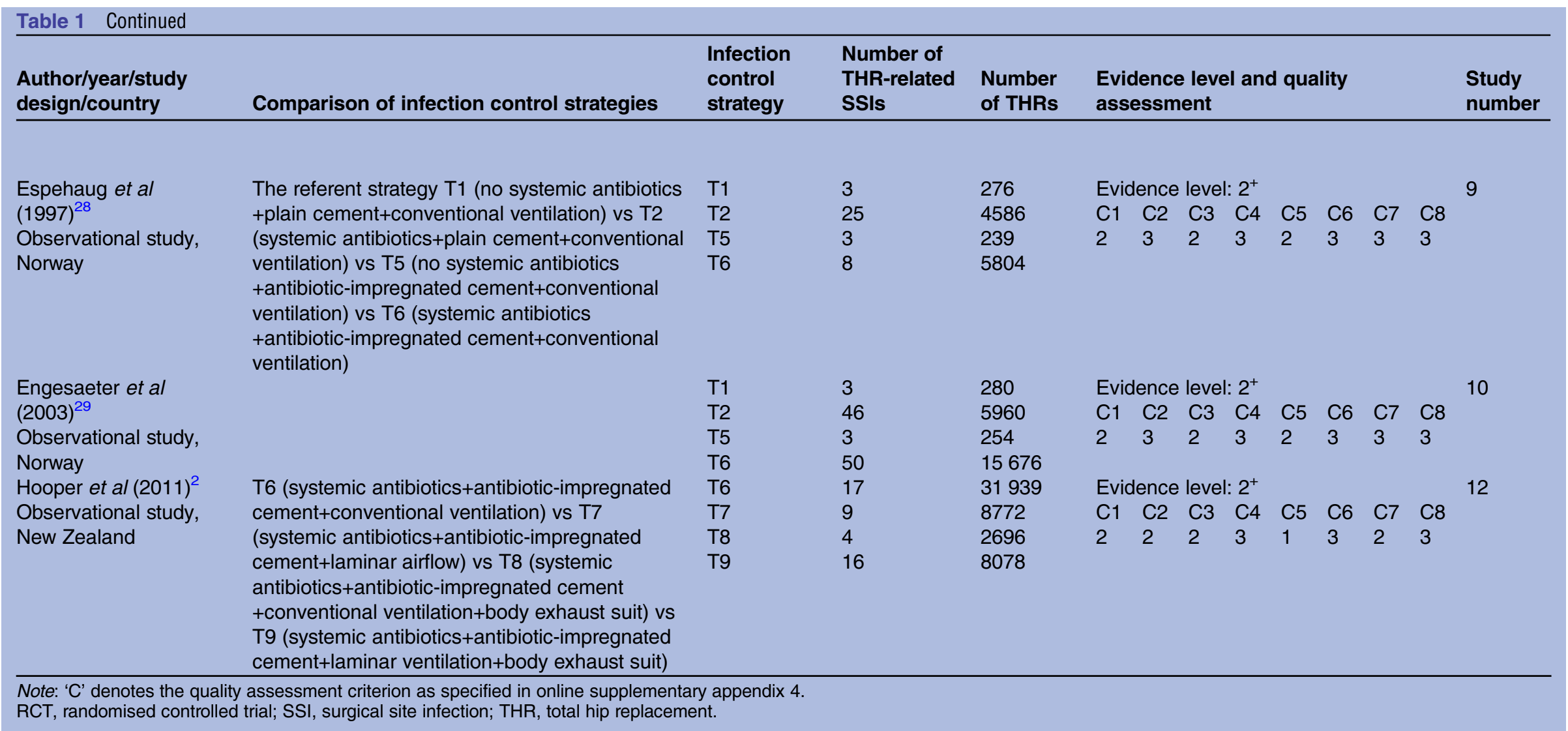


Table 2 ORs with 95\% credible intervals of all nine infection control strategies based on the logit link random effect MTC model

\section{Comparison of infection}

control strategies

OR and $95 \%$ credible interval

\section{OR $(1,2)$}

$0.31(0.12-0.65)$

OR $(1,3)$

OR $(2,3)$

$0.26(0.03-0.95)$

OR $(1,4)$

$0.92(0.11-3.39)$

OR $(2,4)$

OR $(3,4)$

$0.25(0.06-0.66)$

$0.84(0.28-1.97)$

OR $(2,5)$

$1.93(0.20-7.58)$

$1.28(0.38-3.38) \quad 3.28(0.27-14.15)$

$0.38(0.09-1.12)$

OR $(2,6)$

$3.28(0.27-14.15) \quad 1.96(0.37-6.54)$

OR $(1,6)$

$0.13(0.03-0.35)$

OR $(1,7)$

$0.44(0.13-1.13) \quad 1.12(0.09-4.62)$

OR $(2,7) \quad$ OR $(3,7)$

OR $(4,6)$

$0.27(0.03$ to 0.93$)$

OR $(1,8)$

$0.90(0.13-3.14) \quad 2.47(0.11-10.22)$

OR $(2,8) \quad$ OR $(3,8)$

$0.67(0.12-2.12)$

OR $(4,7)$

$1.41(0.14-5.35)$

OR $(4,8)$

$1.77(0.11-7.20) \quad 5.78(0.10-21.12) \quad 2.89(0.12-11.73)$

$1.71(0.08-6.93)$

$\begin{array}{lll}\text { OR }(1,9) & \text { OR }(2,9) & \text { OR }(3,9) \\ 0.74(0.05-2.69) & 2.49(0.20-9.11) & 13.15(0.18-27.4)\end{array}$

$\begin{array}{ll}\mathrm{OR}(4,9) & \mathrm{OR}(5,9) \\ 4.11(0.22-14.92) & 2.44(0.15-8.62)\end{array}$

OR $(5,6)$

$0.43(0.09-1.24)$

OR $(5,7)$

OR $(6,7)$

$0.88(0.09-3.10) \quad 1.96(0.52-5.37)$

OR $(5,8) \quad$ OR $(6,8)$

OR $(6,8) \quad$ OR $(7,8)$

Model fit statistic (posterior mean residual deviance) $34.3^{*}$

$3.72(0.38-13.75) \quad 2.26(0.22-8.48)$

OR $(6,9) \quad$ OR $(7,9)$

OR $(8,9)$

Model fit statistic (DIC) 180.6

Heterogeneity (between-study deviation) 0.63

${ }^{*}$ Compared with 32 data points (model fit is considered to be adequate if the posterior mean residual deviance is approximately equal to the number of total data points; see online supplementary appendix 6 for reference).

DIC, deviance information criterion; MTC, mixed treatment comparison. 


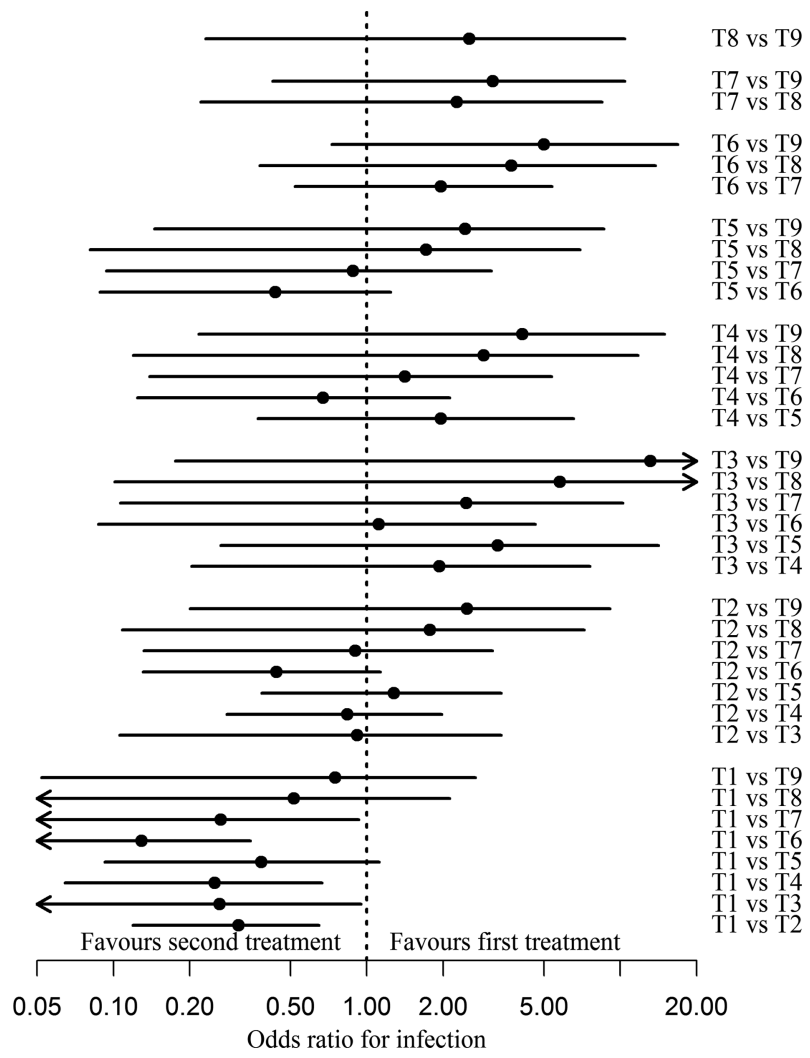

Figure 3 The forest plot of ORs of infection control strategies (random effect model).

T2 and T5 of study 4 and the referent strategy of study 10 were outliers contributing to the inadequate model fit (see online supplementary appendix 13). After exclusion of these two studies, the model fitted the data well, as indicated by model fit statistics, and the heterogeneity was significantly reduced, but the results were little changed (see online supplementary appendix 14). Infection control strategy T6 remained dominant with the highest probability $(64 \%)$ and highest median rank of being the most effective strategy (see online supplementary appendix 15), and OR 0.09 (95\% CrI 0.020.25 ; see online supplementary appendix 14 ).

The direct evidence from all conventional pairwise meta-analyses was presented in online supplementary appendix 16. There was broad agreement among the direct evidence from: conventional pairwise meta-analyses, the direct and indirect evidence from node splitting, and the evidence from the MTC model (see online supplementary appendix 16). Tests for inconsistency between direct and indirect evidence from node splitting suggested that there was no statistically significant evidence of inconsistency. The model fit statistics for the node-splitting and the MTC models were similar, implying that there was no conflict between the direct and indirect evidence (see online supplementary appendix 16). It is worth noting that the $95 \%$ CrIs for some pairwise comparisons widened greatly following node splitting. This is explained by the node splitting reducing the evidence available to inform the variance.
A test of interaction between RCTs and observational studies was not statistically significant, suggesting that combining these study types was not inappropriate (see online supplementary appendix 17).

The results were little changed by excluding the RCT by Hill $e t a 2^{20}$ or including the RCT by Lidwell ${ }^{1}$ et al. Strategy T6 remained dominant with the highest probability (63\% and $83 \%$, respectively) and highest median rank of being the most effective strategy (the details of the sensitivity analyses are shown in online supplementary appendix 18).

\section{DISCUSSION}

Laminar airflow has been widely used as an important infection control measure in many countries around the world. In the UK, for instance, around $98 \%$ of all hip arthroplasties are carried out in operating theatres equipped with laminar airflow systems. ${ }^{30}$ The current infection control guidelines in the $\mathrm{UK}^{31}$ and the $\mathrm{USA}^{32}$ recommend the use of laminar airflow to reduce THR-related SSIs. It is an expensive technology, costing US\$60 000-US\$90000 for construction and installation for each operating room. ${ }^{33}$ However, our study showed that conventional ventilation together with systemic antibiotics and antibiotic-impregnated cement was most likely to provide the best protection against THR-related SSIs. We found no convincing evidence in favour of the use of laminar airflow over conventional ventilation for prevention of THR-related SSIs.

Although the point estimate for the infection control strategy T3 (no systemic antibiotics+plain cement +laminar airflow) compared with the referent was statistically significant, caution needs to be taken in its interpretation because it had only one RCT conducted between 1975 and 1978 by Hills and colleagues, and the study reported that about $8 \%$ of the patients (99 in the placebo group and 70 in the antibiotic group) did not follow the RCT trial protocol with unreported use of antibiotics. We therefore conducted a sensitivity analysis by excluding this RCT from the MTC network and the results for other strategies changed little, with $\mathrm{T} 6$ remaining the most effective.

A recent systematic review concluded that laminar airflow tended to lower infection rates as opposed to conventional ventilation, but the authors emphasised that it was difficult to draw a definite conclusion due to confounding. ${ }^{8}$ The systematic review was descriptive, involving no statistical analysis. It cited, among others, the RCT by Lidwell $e t a l^{1}$ as the key evidence for reducing wound infection using laminar airflow. However, this RCT did not control for antibiotics as a significant confounder. Our sensitivity analysis found that the overall results changed little with the inclusion of this RCT, so it had little influence on our conclusions.

Contrary to the key early evidence in the late 1960s to early 1980s that laminar airflow and body exhaust suit reduced wound contamination and SSIs, ${ }^{1}{ }^{34}$ a number 


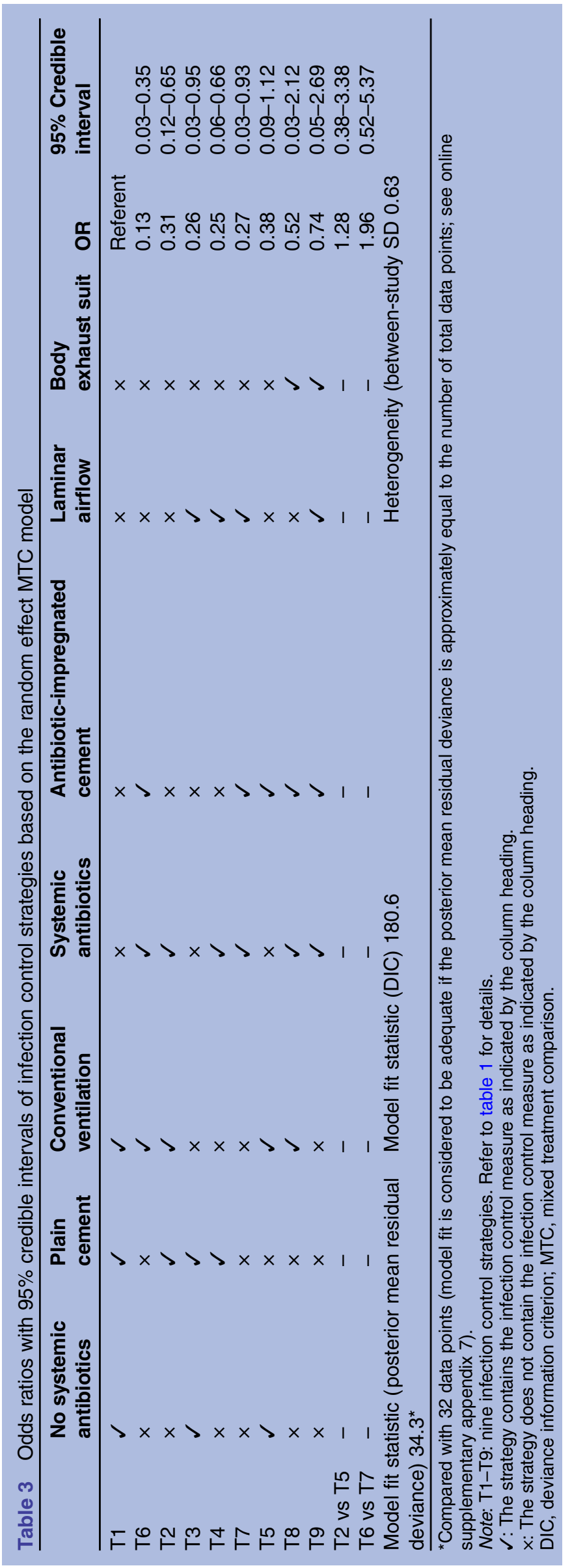

of subsequent studies in the 1980s and 1990s found no convincing evidence that laminar airflow was effective in reducing SSIs compared with conventional ventilation. ${ }^{2326}{ }^{27}$ Four recent large-scale observational studies of 113183 THRs suggested that laminar airflow and body exhaust suit conferred no protective benefit against SSI and might increase the risk of SSI. ${ }^{2} 32829$

A hypothesis of the early studies by Charnley ${ }^{34}$ and Lidwell et $a l^{1}$ on laminar airflow and body exhaust suits was that one of the main routes of wound contamination and infection was the air in the operating room, and laminar airflow and body exhaust suits could reduce airborne bacteria load and therefore wound contamination and infection. The studies demonstrated that laminar airflow ventilation reduced airborne organisms or colony forming units (cfu) to $10 \mathrm{cfu} / \mathrm{m}^{3}$ between 150 and $300 \mathrm{cfu} / \mathrm{m}^{3}$ in conventional operating theatres. To be most effective, key operating theatre staff should wear body exhaust suits while working in the ultraclean environment. ${ }^{134}$ However, standard culture techniques by air sampling or landing 'mode' as a method to assess potential wound contamination do not directly correlate with wound contamination, and they are at best surrogate measures representing the degree of air contamination at the point of sampling, which might be some distance away from the implant zone. ${ }^{35}$ There was also evidence that wound contamination was greater at the end of surgery than at the beginning. ${ }^{35}$

The surgeons' heads position above the surgical site and directly in the laminar airstream might facilitate pathogen-contaminating particles falling directly into the wound. ${ }^{36}$ Laminar airflow could also result in lower intraoperative tissue temperatures in the surgical wound, ${ }^{3}$ and systemic hypothermia is a known risk factor for SSI. ${ }^{37}$

Our study found no high-quality evidence that antibiotic-impregnated cement without systemic antibiotic prophylaxis was effective in reducing THR-related SSI compared with plain cement with systemic antibiotic prophylaxis. Contrary to our findings, a recent meta-analysis showed that the use of antibioticimpregnated cement lowered the infection rate by approximately $50 \%$ compared with plain cement. ${ }^{6}$ However, the meta-analysis failed to stratify the infection control arms according to antibiotic regimens and pool on a comparable basis. We reanalysed the data from this meta-analysis by stratifying infection control arms based on antibiotic regimens and pooling the rest of the studies on a comparable basis for summary estimation (see online supplementary appendix 19). The pooled relative risk of antibiotic-impregnated cement compared with plain cement was 0.76 (95\% CI 0.46 to 1.28$)$. So there was no high-quality evidence that antibiotic-impregnated cement without systemic antibiotic prophylaxis was effective in reducing THR-related infection compared with plain cement with systemic antibiotic prophylaxis.

A RCT showed that antibiotic-impregnated cement together with systemic antibiotic prophylaxis was 
effective in reducing knee replacement-related infection compared with plain cement with systemic antibiotic prophylaxis. ${ }^{38}$ All the procedures were performed in a standard operating room without laminar airflow or body-exhaust suit. The authors stressed that while they did not believe that antibiotic-impregnated cement alone would prevent deep infection, it could aid in prevention of early or intermediate infection in conjunction with systemic antibiotic prophylaxis. This might be explained by the capacity of antibiotic-impregnated cement as a drug-delivery vehicle. It was suggested that the polymeric nature of polymethylmethacrylate allowed ingress of physiological fluids, which permitted elution of incorporated antibiotic, but the relative hydrophobicity of bone cement allowed only $10 \%$ of the antibiotic to elute effectively. ${ }^{39}$

Our evidence synthesis has limitations. The small number of studies available for evidence synthesis reduced the statistical power and resulted in wide CrIs for some comparisons. MTC can only synthesise evidence in a connected network. Consequently, one study $^{40}$ meeting our inclusion criteria could not be included as it could not be connected to the network. However, the exclusion of this study should not change our results, as the study concluded that there was no statistical difference in THR-related SSIs between plain cement and antibiotic-impregnated cement, which accorded with our findings.

Owing to the limited data available, the MTC model was unable to adjust for potential confounders such as case-mix, particularly patient comorbidity in different hospital settings, different types of laminar airflow systems used (eg, horizontal vs vertical laminar airflow systems), and temporal changes in clinical practices, infection control technology (eg, the use of ultra-high flows in modern conventional operating theatres and forced air blankets) and patient profiles which may have taken place over the past several decades.

The evidence in our study covered a period from 1966 up to June 2011 when the literature search was performed. The evidence needs to be updated when new studies become available.

\section{CONCLUSIONS}

This is the first study to examine the comparative effectiveness of various infection control strategies involving multiple infection control measures in preventing THR-related SSI. We found no convincing evidence in favour of the use of laminar airflow over conventional ventilation for prevention of THR-related SSI. Systemic antibiotic prophylaxis in conjunction with antibioticimpregnated cement and conventional ventilation was likely to be the most effective infection control strategy for preventing THR-related SSI based on the available evidence. There was no high-quality evidence that antibiotic-impregnated cement alone without systemic antibiotic prophylaxis was effective in reducing
THR-related SSI compared with plain cement with systemic antibiotics. Our evidence synthesis underscores the need to review current guidelines based on the available evidence, and to conduct further high-quality doubleblind RCTs to better inform the current clinical guidelines and practice for prevention of THR-related SSI.

Contributors NG, AGB, HZ, KM, AS, NC, TB and JW were involved in the inception and design of the study. NG and $A G B$ oversaw the implementation of the study. $\mathrm{HZ}$ and $\mathrm{KM}$ were involved in study identification and data acquisition. JW advised on interpretation of the literature. $\mathrm{HZ}$ performed the statistical analysis with help from AGB. HZ, AGB, NG, AS, NC,TB and JW interpreted the data. $\mathrm{HZ}$ wrote the first draft of the paper with input from AGB and NG. AGS, NC, TB and JW critically reviewed and revised the draft. All authors approved the final version of the manuscript for publication.

Funding The project was funded by the UK National Institutes for Health Research and the Queensland Health Quality Improvement and Enhancement Programme (grant number 2008001769).

Competing interests None.

Provenance and peer review Not commissioned; externally peer reviewed.

Data sharing statement The relevant data and codes used in this study are available from the authors.

Open Access This is an Open Access article distributed in accordance with the Creative Commons Attribution Non Commercial (CC BY-NC 3.0) license, which permits others to distribute, remix, adapt, build upon this work noncommercially, and license their derivative works on different terms, provided the original work is properly cited and the use is non-commercial. See: http:// creativecommons.org/licenses/by-nc/3.0/

\section{REFERENCES}

1. Lidwell OM, Lowbury EJ, Whyte W, et al. Effect of ultraclean air in operating rooms on deep sepsis in the joint after total hip or knee replacement: a randomised study. BMJ (Clin Res Ed) 1982;285:10-14.

2. Hooper GJ, Rothwell AG, Frampton C, et al. Does the use of laminar flow and space suits reduce early deep infection after total hip and knee replacement?: the ten-year results of the New Zealand Joint Registry. J Bone Joint Surg Br 2011;93:85-90.

3. Brandt $\mathrm{C}$, Hott $\mathrm{U}$, Sohr D, et al. Operating room ventilation with laminar airflow shows no protective effect on the surgical site infection rate in orthopedic and abdominal surgery. Ann Surg 2008;248:695-700.

4. Glenny A, Song F. Antimicrobial prophylaxis in total hip replacement: a systematic review. Health Technol Assess (Winchester, England) 1999;3:1-57.

5. AlBuhairan B, Hind D, Hutchinson A. Antibiotic prophylaxis for wound infections in total joint arthroplasty: a systematic review. J Bone Joint Surg Br 2008;90:915-19.

6. Parvizi J, Saleh KJ, Ragland PS, et al. Efficacy of antibiotic-impregnated cement in total hip replacement. Acta Orthop 2008;79:335-41.

7. Block JE, Stubbs HA. Reducing the risk of deep wound infection in primary joint arthroplasty with antibiotic bone cement. Orthopedics 2005;28:1334-45.

8. Whitehead S, Bending M, Trueman P, et al. Cost-effectiveness of hospital design: options to improve patient safety and wellbeing: systematic literature review of ventilation. York: York Health Economics Consortium, 2008.

9. Sutton A, Ades AE, Cooper N, et al. Use of indirect and mixed treatment comparisons for technology assessment. Pharmacoeconomics 2008;26:753-67.

10. Caldwell DM, Ades AE, Higgins JPT. Simultaneous comparison of multiple treatments: combining direct and indirect evidence. $B M J$ (Clin Res Ed) 2005;331:897-900.

11. Merollini KMD, Zheng H, Graves N. Most relevant strategies for preventing surgical site infection after total hip arthroplasty: guideline recommendations and expert opinion. Am J Infect Control 2013;41:221-6.

12. Moher D LA, Tetzlaff J, Altman DG, The PRISMA Group. Preferred reporting items for systematic reviews and meta-analyses: the PRISMA statement. 2009. http://www.prisma-statement.org/ statement.htm 
13. National Institute for Health and Clinical Excellence. Methods for development of NICE public health guidance. http://www.nice.org.uk/ page.aspx?o=299970 (accessed 16 May 2010)

14. The Joanna Briggs Institute. Joanna Briggs Institute Reviewer's Manual:2008 edition. 2008.

15. Tooth L, Ware R, Bain C, et al. Quality of reporting of observational longitudinal research. Am J Epidemiol 2005;161:280-8.

16. Lu G, Ades AE. Combination of direct and indirect evidence in mixed treatment comparisons. Stat Med 2004;23:3105-24.

17. Dias S, Welton NJ, Sutton AJ, et al. NICE DSU Technical Support Department 2: a generalized linear modelling framework for pair-wise and network meta-analysis. 2011. http://www.nicedsu.org. uk (accessed 7 Jan 2012)

18. Dias S, Welton NJ, Caldwell DM, et al. Checking consistency in mixed treatment comparison meta-analysis. Stat Med 2010;29:932-44.

19. Dias S, Welton NJ, Sutton AJ, et al. NICE DSU Technical Support Department 3: heterogeneity, meta-regression, bias and bias adjustment. 2011. http://www.nicedsu.org.uk (accessed 7 Jan 2012).

20. Hill C, Flamant R, Mazas F, et al. Prophylactic cefazolin versus placebo in total hip replacement. Report of a multicentre double-blind randomised trial. Lancet 1981;1:795-6.

21. Carlsson AK, Lidgren L, Lindberg L. Prophylactic antibiotics against early and late deep infections after total hip replacements. Acta Orthop Scand 1977;48:405-10.

22. Schulitz KP, Winkelmann W, Schoening B. The prophylactic use of antibiotics in alloarthroplasty of the hip joint for coxarthrosis. Arch Orthop Trauma Surg 1980;96:79-82.

23. Fitzgerald RH Jr. Total hip arthroplasty sepsis. Prevention and diagnosis. Orthop Clin North Am 1992;23:259-64.

24. Josefsson G, Lindberg L, Wiklander B. Systemic antibiotics and gentamicin-containing bone cement in the prophylaxis of postoperative infections in total hip arthroplasty. Clin Orthop Relat Res 1981;159:194-200.

25. McQueen MM, Hughes SP, May P, et al. Cefuroxime in total joint arthroplasty. Intravenous or in bone cement. $J$ Arthroplasty 1990;5:169-72.

26. Salvati EA, Robinson RP, Zeno SM, et al. Infection rates after 3175 total hip and total knee replacements performed with and without a horizontal unidirectional filtered air-flow system. J Bone Joint Surg Am 1982;64:525-35

27. Kelly AJ, Bailey R, Davies EG, et al. An audit of early wound infection after elective orthopaedic surgery. J Royal Coll Surg Edinb 1996;41:129-31.
28. Espehaug B, Engesaeter LB, Vollset SE, et al. Antibiotic prophylaxis in total hip arthroplasty. Review of 10,905 primary cemented total hip replacements reported to the Norwegian arthroplasty register, 1987 to 1995. J Bone Joint Surg Br 1997;79:590-5.

29. Engesaeter LB, Lie SA, Espehaug B, et al. Antibiotic prophylaxis in total hip arthroplasty: effects of antibiotic prophylaxis systemically and in bone cement on the revision rate of 22,170 primary hip replacements followed 0-14 years in the Norwegian Arthroplasty Register. Acta Orthop Scand 2003;74:644-51.

30. Walenkamp GHIM. Joint prosthetic infections: a success story or a continuous concern? Acta Orthop 2009;80:629-32.

31. British Orthopaedic Association. Primary total hip replacement: a guide to good practice. London: British Orthopaedic Association, 2006.

32. Mangram AJ, Horan TC, Pearson ML, et al. Guideline for prevention of surgical site infection, 1999. Centers for Disease Control and Prevention (CDC) Hospital Infection Control Practices Advisory Committee. Am J Infect Control 1999;27:97.

33. Evans RP. Current concepts for clean air and total joint arthroplasty: laminar airflow and ultraviolet radiation: a systematic review. Clin Orthop Relat Res 2011;469:945-53.

34. Charnley J. Postoperative infection after total hip replacement with special reference to air contamination in the operating room. Clin Orthop Relat Res 1972;87:167-87.

35. Clarke MT, Lee PTH, Roberts CP, et al. Contamination of primary total hip replacements in standard and ultra-clean operating theaters detected by the polymerase chain reaction. Acta Orthop Scand 2004;75:544-8.

36. Hubble MJ, Weale AE, Perez JV, et al. Clothing in laminar-flow operating theatres. J Hosp Infect 1996;32:1-7.

37. Melling AC, Ali B, Scott EM, et al. Effects of preoperative warming on the incidence of wound infection after clean surgery: a randomised controlled trial. Lancet 2001;358:876-80.

38. Chiu F-Y, Chen C-M, Lin C-FJ, et al. Cefuroxime-impregnated cement in primary total knee arthroplasty: a prospective, randomized study of three hundred and forty knees. J Bone Joint Surg Am 2002;84-A:759-62.

39. DiCicco M, Duong T, Chu A, et al. Tobramycin and gentamycin elution analysis between two in situ polymerizable orthopedic composites. J Biomed Mater Res B Appl Biomater 2003;65:137-49.

40. Lynch M, Esser MP, Shelley $P$, et al. Deep infection in Charnley low-friction arthroplasty. Comparison of plain and gentamicin-loaded cement. J Bone Joint Surg Br 1987;69:355-60. 